\title{
Intradural Extramedullary Spinal Ependymoma: A Case Report of Malignant Transformation Occurring
}

\author{
Takashi Moriwaki, Koichi Iwatsuki, Yu-ichiro Ohnishi, \\ Masao Umegaki, Masahiro Ishihara ,Toshiki Yoshimine \\ Department of Neurosurgery, Osaka University Graduate School of Medicine, Suita, Japan
}

\begin{abstract}
Intradural extramedullary spinal ependymomas are extremely rare. Herein, we describe a lesion-type spinal ependymoma that followed a malignant course, and discuss its clinical presentation, etiopathogenesis, and treatment. We present a patient who was diagnosed with an intradural extramedullary spinal tumor at T4-T6. The patient underwent gross total resection of the tumor without damage to the spinal cord. Histological examination, classified the lesion as a World Health Organization (WHO)-grade 2 ependymoma. One and a half years later, magnetic resonance imaging detected a recurring tumor at T4-T5. The tumor was removed and classified as a WHO-grade 3 anaplastic ependymoma. The patient was started on a course of regional spinal cord radiotherapy. The patient achieved tumoral control and clinical stabilization after the recurrence. We must consider the differential diagnosis of intradural extramedullary spinal tumors. The best treatment for this lesion is gross total resection and adjunctive radiotherapy is necessary in cases of malignant-change.
\end{abstract}

Keywords: Ependymoma; Intradural extramedullary spinal cord neoplasms; Spinal cord tumor

\section{Introduction}

Spinal ependymomas are the most common intramedullary tumors and occur predominantly in adults. Other than those located at the terminal filum or conus medullaris, intradural extramedullary ependymomas are extremely rare [1]. Ependymomas arising outside the lesion of the conus medullaris, cauda equina, and terminal filum, or developing from ectopic ependymal cells are highly unusual $[2,3]$.

Although these tumors are most often benign, a few may follow a more malignant course. Anaplastic transformation and cerebrospinal fluid dissemination rarely occur $[2,3]$.

We describe a rare case and malignant course of intramedullary ependymoma and discuss its clinical presentation, etiopathogenesis, and treatment.

\section{Case Report}

A 23-year-old woman was admitted to our institution on January 2009. Her symptoms included a backache around her right shoulder, paresthesia in the left lower limb, pain in the left abdominal region, and difficulty walking smoothly (as a result of the backache and pain). The patient had first noticed the backache 4 months be-

Received Dec 24, 2011; Revised Mar 6, 2012; Accepted Apr 2, 2012

Corresponding author: Takashi Moriwaki

Department of Neurosurgery, Osaka University Graduate School of Medicine,

2-2 Yamadaoka, Suita 565-0871, Japan

Tel: +81-6-6879-3652, Fax: +81-6-6879-3659, E-mail: t-moriwaki@nsurg.med.osaka-u.ac.jp 
fore admission, and her neurological status progressively worsened.

Neurological examination revealed slightly motor weakness of left lower limb. She had sensory disturbances of above. Examinations were negative for the Romberg sign, with bilateral Babinski's sign. Deep tendon reflexes were nearly symmetrical in the lower extremities. Urinary function, rectal tone, and perianal sensation were normal.

Cranial computed tomography (CT), and abdominal, chest X-ray were within normal range, magnetic resonance imaging (MRI) of the thoracic spine (Fig. 1) showed a posterolateral intradural extramedullary mass compressing the spinal cord at the T4-T6 levels. The mass was of low intensity in medullary tissue for T1 imaging and of slightly high intensity in the T2 sequence, with homogeneous enhancement after gadolinium injection. Its limits were well defined, occupying $60 \%$ of the posterior and left spinal canal without bone destruction or foramen invasion, suggesting schwannoma or meningioma as the first option. No other lesions were present along the neuroaxis, including the posterior fossa.

Because the tumor was present in the left spinal ca-
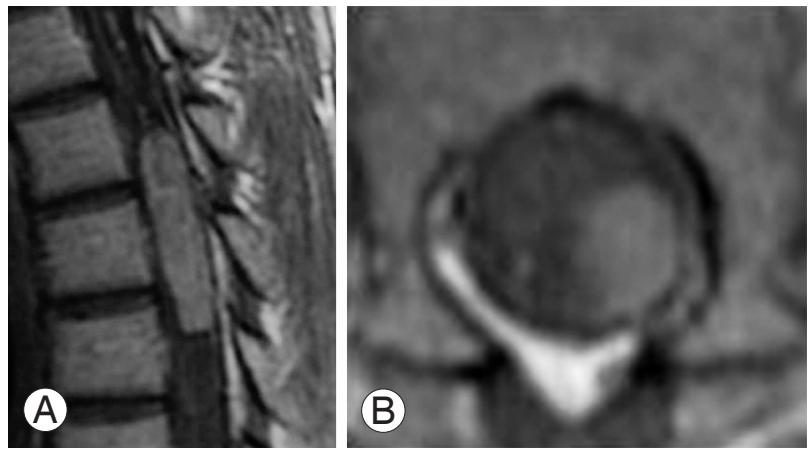

Fig. 1. Sagittal T1-weighted, gadolinium-enhanced (A), axial T1weighted, gadolinium-enhanced (B) views of preoperative magnetic resonance imaging (3.0T) displaying the posterolateral intradural extramedullary T4-T6 ependymoma with spinal cord compression displaced ventrally. nal, a left hemilaminectomy from T4 to T6 (Fig. 2) was performed, uncovering an intradural extramedullary encapsulated mass. We determined that the tumor was not connected to the dura mater or spinal roots, and had not infiltrated the spinal cord. Only mild adhesions to the spinal pia mater were found and were carefully stripped away.

A gross total resection was completed without damage to the medulla, using an ultrasonic surgical aspirator under an operating microscope.

Anatomic-pathologic studies were performed. Immunohistochemical examination (Fig. 3) demonstrated strong immunopositivity with anti-glial fibrillary acidic protein, especially in perivascular pseudorosettes, but there was no immunostaining with the epithelial membrane antigen. Neither cystic degeneration nor anaplastic change was present, but the Ki-67 index was 5\% to $10 \%$.

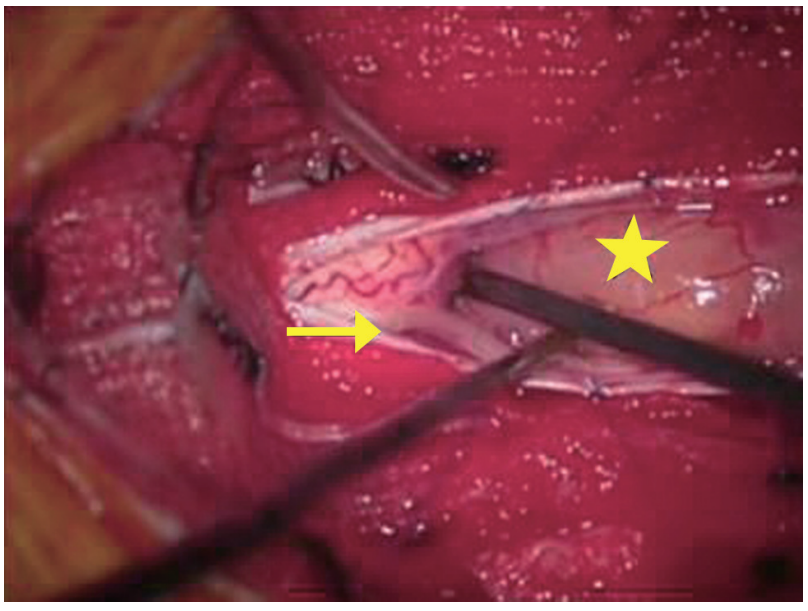

Fig. 2. A posterior T4-T6, left hemilaminectomy allowed intradural exploration and removal of the intradural extramedullary mass (star). A reasonable dissecting plane was discerned against the spinal cord, and the tumor was extramedullary with partial adhesion to the posterior spinal cord; however, there was no adhesion to either posterior nerve roots of the T4 (arrow). The tumor was excised in gross total fashion using standard microsurgical techniques.
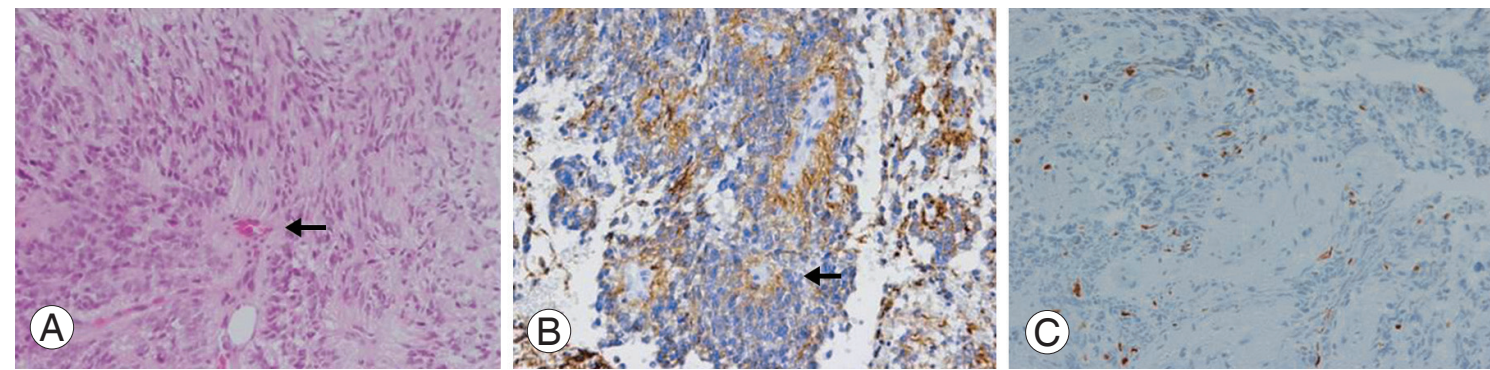

Fig. 3. (A) The histological features of the ependymoma were observed; perivascular pseudorosettes (arrow) were identified (H\&E, $\times 200$ ). (B) GFAP immunoreactivity was observed predominantly around tumor vessels (arrow) (GFAP immunostain, $\times 200)$. (C) The tumor exhibited slightly high proliferative activity ( $5 \%$ to $10 \%$ ) (Ki-67 immunostain, $\times 100)$. 
Histological examination revealed a World Health Organization (WHO)-grade 2 ependymoma with hypercellular nodules that demonstrated a high Ki-67 index.

The patient's neurologic condition improved after surgery and she was discharged from the hospital with mild paresthesia and pain in the left abdominal region. Neither radiotherapy nor chemotherapy was performed. We carried out close follow-up by MRI (Fig. 4).

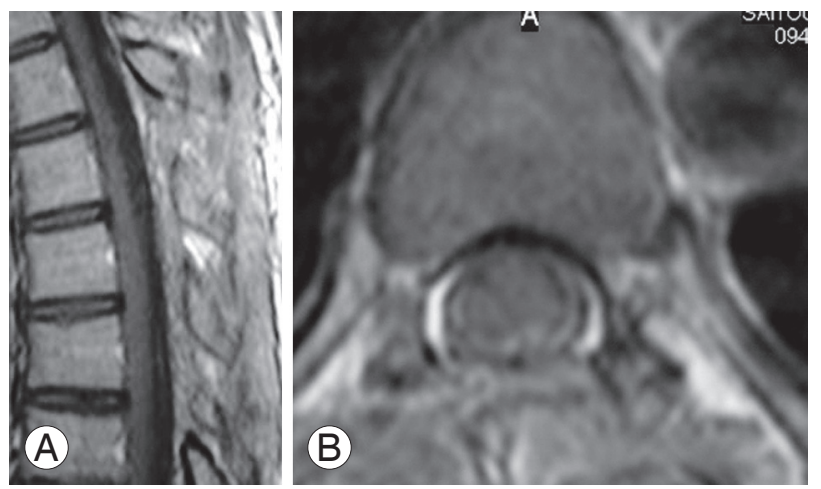

Fig. 4. Sagittal T1-weighted, gadolinium-enhanced (A) and axial T1weighted, gadolinium-enhanced (B) views of first postoperative magnetic resonance images (3.0T), which displayed no tumor recurrence.
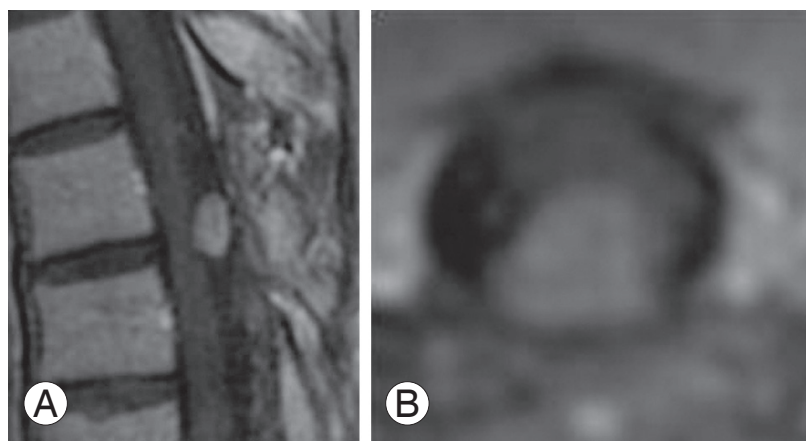

Fig. 5. Sagittal T1-weighted, gadolinium-enhanced (A) and axial T1weighted, gadolinium-enhanced (B) views of recurrence magnetic resonance images (3.0T) displaying the posterior intradural extramedullary T4-T5 anaplastic ependymoma.
One and a half years later, the patient's physical and neurological condition had worsened, with increased pain in the left abdominal region. MRI of the thoracic spine (Fig. 5) uncovered a recurring mass at the T4-T5 levels: a posterior intradural extramedullary tumor.

A T4-T6 laminectomy (Fig. 6) was performed; the tumor was removed and classified as a WHO-grade3 anaplastic ependymoma (Fig. 7). The patient was started on a course of regional spinal cord radiotherapy, which was $50.4 \mathrm{~Gy} / 28 \mathrm{Fr}$. The symptoms in the left abdominal region decreased. Neither worsening nor deterioration was observed after surgery and radiotherapy treatment, with the patient achieving tumoral control and clinical stabilization with a total follow-up of 17 months after the recurrence.

\section{Discussion}

Spinal ependymomas are typically intradural intramedullary tumors, and are often described as benign tumors $[4,5]$. Spinal intradural extramedullary ependymomas are rare [3,5-7]. There have only be 15 cases presented in the

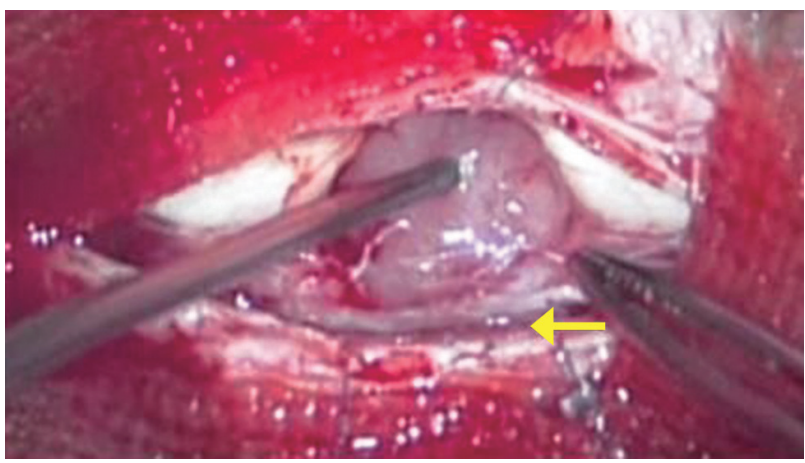

Fig. 6. A posterior T4-T6 total hemilaminectomy was performed and the intradural extramedullary tumor was removed. The tumor had adhered more strongly to the posterior spinal cord than before. However, as before, the tumor had not adhered (arrow) to the posterior nerve roots.
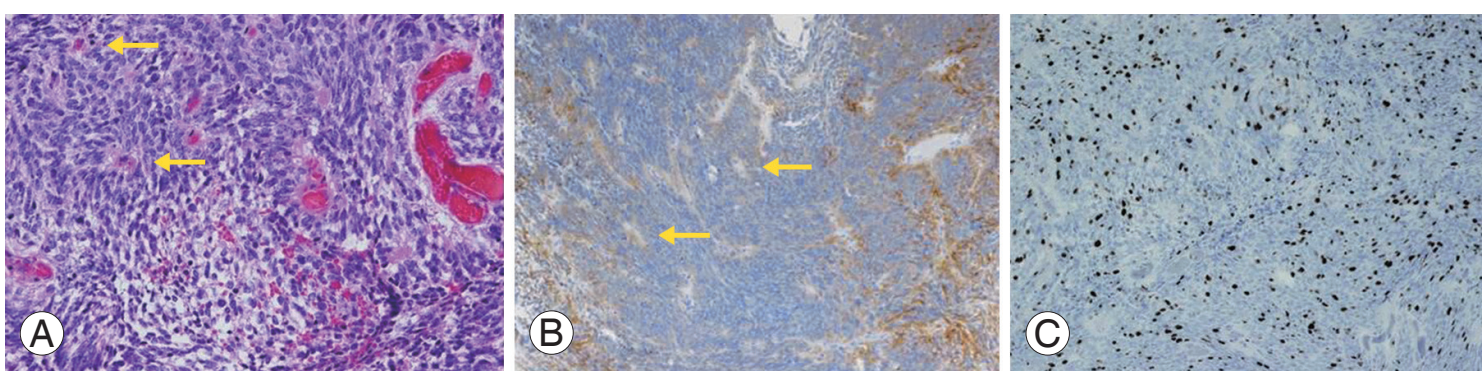

Fig. 7. (A) Yellow arrows indicate densely cellular glial neoplasms with abundant perivascular pseudorosettes characteristic of an ependymoma (H\&E, $\times 200)$. (B) GFAP immunoreactivity was observed predominantly around perivascular pseudorosettes (arrows) (GFAP immunostain, $\times 100)$. (C) The tumor exhibited significantly high proliferative activity (10\% to 15\%) (Ki-67 immunostain, $\times 100)$. 
literature [5].

This lesion-type tumor is believed to arise from ectopic ependymal cell rests. Some ependymal cells may remain on the inside during neural tube closure [8].

In all cases, these types of ependymomas were encapsulated with only microvascular attachment to the spinal cord, and could be removed completely. However, there was mild adhesion to the spinal pia mater in the present case. After complete resection, we carried out close follow-up because of the potential for recurrence, metastasis, and anaplastic transformation.

Although ependymomas are most often benign [9], there have been 3 previous reports of malignant transformation occurring $[1,2,10]$. In this case, histological examination of the second operation identified malignant transformation. This malignant transformation may have led to the recurrence. However, second operative views showed that the recurring ependymoma was more strongly adhered to the spinal pia mater than that of the first views. In the first operation, there was slight adhesion between the spinal pia mater and the encapsulated tumor by a tumor membrane that was unconnected to the posterior spinal roots, spinal cord, and spinal pia, and dura mater. This patient underwent gross-total resection of a posterolateral tumor; however, there was an absence of medullary contusion to prevent surgical complication at the adhesion lesion, which was slight. This slight lesion would have retained a few ependymoma tumor cells, and these cells might have contained malignant components. During the close follow-up periods, some of these lesion cells underwent a malignant change, infiltrated the spinal pia mater, and the posterior spinal cord. As a result, these changes led to tumor recurrence. Tumor recurrence would not have occurred after the first operation if a part of the adhesion lesion had not contained ependymoma tumor cells, or if those cells had not undergone malignant change. Therefore, the few ependymoma cells remaining after the first operation might have adhered to the spinal pia mater, undergone anaplastic change, and carried out meningeal infiltration, which means that the possibility of tumor cell remnants remaining in the area cannot be ruled out.

The best treatment for an extramedullary ependymoma is gross total resection, as was carried out in this case; adjunctive radiotherapy is necessary in malignant-change cases. The extent of resection and the presence of men- ingeal infiltration appear to be very important factors of patient prognosis.

\section{Conflict of Interest}

No potential conflict of interest relevant to this article was reported.

\section{References}

1. Katoh S, Ikata T, Inoue A, Takahashi M. Intradural extramedullary ependymoma: a case report. Spine (Phila Pa 1976) 1995;20:2036-8.

2. Cerase A, Venturi C, Oliveri G, De Falco D, Miracco C. Intradural extramedullary spinal anaplastic ependymoma. Case illustration. J Neurosurg Spine 2006;5:476.

3. Robles SG, Saldana C, Boto GR, et al. Intradural extramedullary spinal ependymoma: a benign pathology? Spine (Phila Pa 1976) 2005;30:E251-4.

4. Graca J, Gultasli N, D’Haene N, Brotchi J, Salmon I, Baleriaux D. Cystic extramedullary ependymoma. AJNR Am J Neuroradiol 2006;27:818-21.

5. Kinsman MJ, Callahan JD, Hattab EM, Cohen-Gadol AA. Extramedullary spinal ependymoma: a diagnostic challenge and review of the literature. Clin Neurol Neurosurg 2011;113:661-4.

6. Duffau H, Gazzaz M, Kujas M, Fohanno D. Primary intradural extramedullary ependymoma: case report and review of the literature. Spine (Phila $\mathrm{Pa} 1976$ ) 2000;25:1993-5.

7. Iunes EA, Stavale JN, de Cassia Caldas Pessoa R, et al. Multifocal intradural extramedullary ependymoma. Case report. J Neurosurg Spine 2011;14:65-70.

8. Benzagmout M, Boujraf S, Oulali N, et al. Intradural extramedullary ependymoma: is there constantly a hormonal relationship? Surg Neurol 2008;70:536-8.

9. Kaner T, Sasani M, Oktenoglu T, Solmaz B, Sarloglu AC, Ozer AF. Clinical analysis of 21 cases of spinal cord ependymoma: positive clinical results of gross total resection. J Korean Neurosurg Soc 2010;47:1026.

10. Schuurmans M, Vanneste JA, Verstegen MJ, van Furth WR. Spinal extramedullary anaplastic ependymoma with spinal and intracranial metastases. J Neurooncol 2006;79:57-9. 